Musées, Patrimoine et Culture scientifiques et techniques

$166 \mid 2016$

juillet-août 2016

\title{
Quel rôle pour les muséums dans la production et la diffusion de la connaissance naturaliste à l'époque d'Internet?
}

\section{Catherine Gauthier}

\section{OpenEdition}

\section{Journals}

Édition électronique

URL : http://journals.openedition.org/ocim/1669

DOI : 10.4000/ocim.1669

ISSN : 2108-646X

Éditeur

OCIM

Édition imprimée

Date de publication : 1 juillet 2016

Pagination : 5-12

ISSN : 0994-1908

\section{Référence électronique}

Catherine Gauthier, «Quel rôle pour les muséums dans la production et la diffusion de la

connaissance naturaliste à l'époque d'Internet? », La Lettre de I'OCIM [En ligne], 166 | 2016, mis en ligne le 01 juillet 2017, consulté le 01 mai 2019. URL : http://journals.openedition.org/ocim/1669 ; DOI

10.4000/ocim. 1669

Ce document a été généré automatiquement le 1 mai 2019.

Tous droits réservés 


\section{Quel rôle pour les muséums dans la production et la diffusion de la connaissance naturaliste à l'époque d'Internet?}

Catherine Gauthier

Nature-Isère, une communauté organisée autour de la connaissance de la nature.

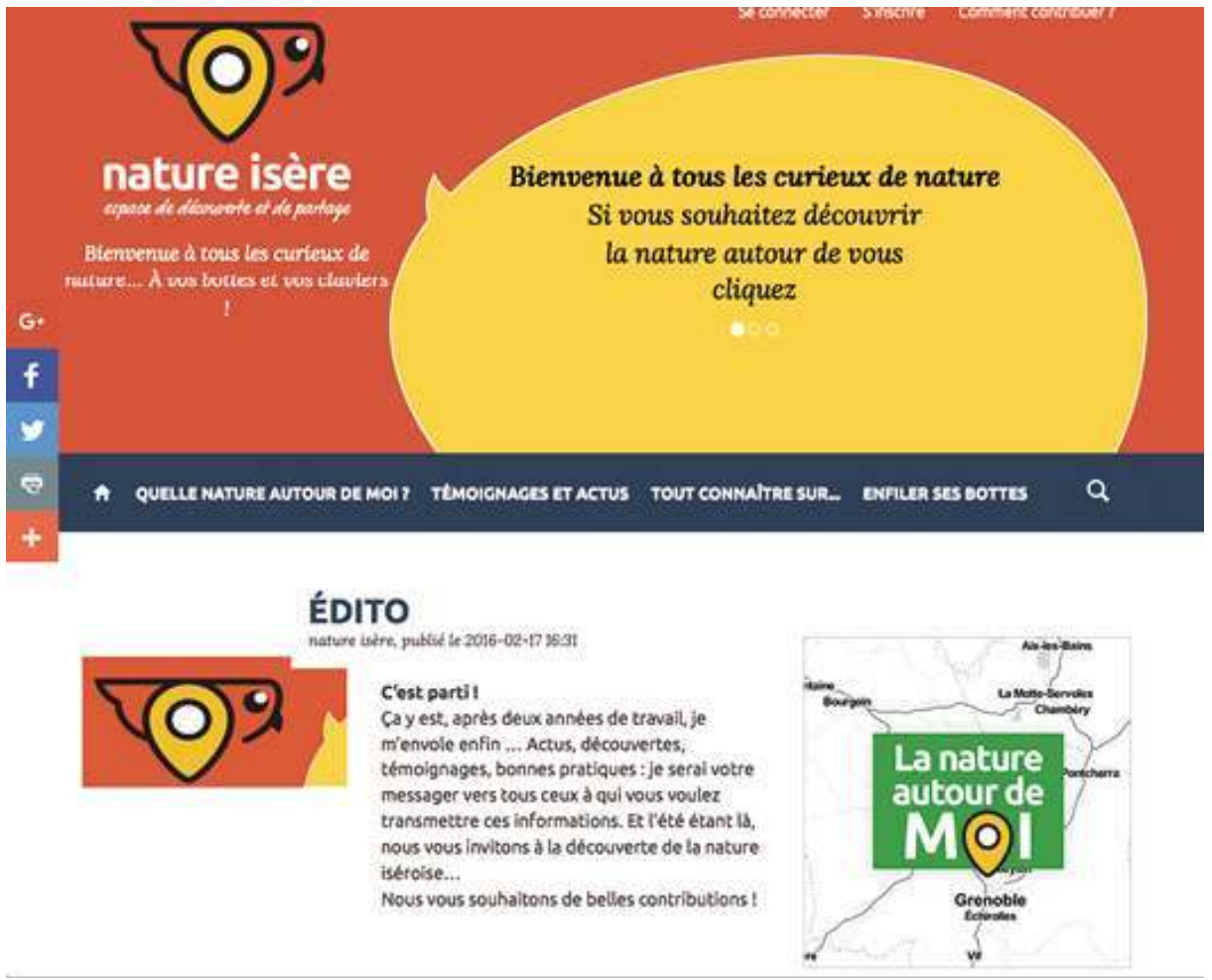

(c) Muséum de Grenoble 
1 En Isère, l'axe 1 du Schéma départemental des espaces naturels sensibles prévoyait depuis 2008 de développer la connaissance des espèces et des milieux naturels présents localement, notamment au bénéfice des gestionnaires de ces espaces et d'une sensibilisation du grand public. Néanmoins, le projet achoppait car aucun acteur n'en avait véritablement pris la coordination. Par ailleurs, l'ambition de mener des campagnes d'inventaire sur tout le territoire isérois poussait l'ensemble des acteurs à vouloir développer le réseau des observateurs de terrain.

2 En 2013, le muséum de Grenoble a donc proposé la mise en commun de toutes les connaissances et des bonnes pratiques liées à l'observation du monde naturel en Isère, pour le rendre accessible à tous via une ligne éditoriale adaptée. Une étude stratégique a préconisé la conception d'un espace éditorial contributif ouvert, en s'appuyant sur une plateforme de données existante et un réseau extrêmement dynamique d'acteurs, y compris aux niveaux numérique et éducatif.

3 Cet article se propose tout d'abord de présenter les risques liés au lancement de tels projets numériques de production de la connaissance en crowdsourcing. Il explique ensuite comment un muséum, institution publique engagée de manière bienveillante auprès de tous, peut mettre en mouvement un tel réseau autour de la coproduction d'un espace éditorial numérique commun, incarnation d'une "communauté" d'intérêt regroupant des acteurs comme des enseignants, des agriculteurs, des gestionnaires d'espaces naturels et des jeunes impliqués dans des projets d'observation. Enfin, l'article s'attache à décrire en quoi la communauté Nature-Isère est originale, à savoir qu'elle a pour objectif premier de créer de l'émulation collective et de renforcer l'estime de soi des contributeurs, délivrant une image positive de ceux qui contribuent et ainsi inciter de plus en plus de citoyens à agir pour la connaissance du monde naturel. 
Pour l'exposition Monstrueux, vous trouvez ça normal ? réalisée en collaboration avec La Casemate, le muséum de Grenoble a développé un projet de partage de contenus sur la plateforme Echosciences Grenoble.

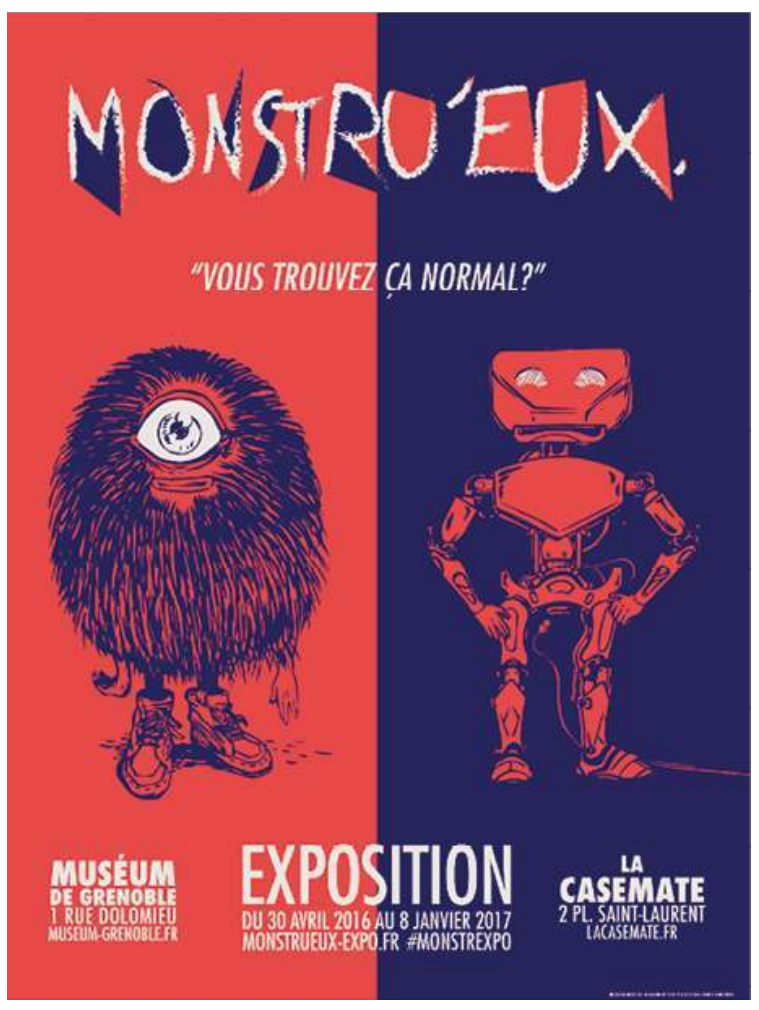

(c) Muséum de Grenoble

\section{Deux siècles de diffusion de la connaissance naturaliste}

\section{Avant le numérique}

$\mathrm{Au} \mathrm{XVIII}^{\mathrm{e}}$ siècle, la construction collective du savoir a été possible grâce aux premières revues scientifiques et aux riches correspondances des savants qui n'hésitent pas à faire le "Grand Tour" de l'Europe pour visiter des cabinets de curiosités, rencontrer leurs homologues et ramener de leurs périples de nombreux livres et manuscrits. L'importance des communautés dans la production et la diffusion du savoir s'incarne dans la multiplication des académies puis des sociétés savantes (1772, création de l'Académie Delphinale de Grenoble). Elles sont remplacées durant la Révolution française par des sociétés savantes spécialisées quasi "professionnelles": l'observation et l'expérimentation sont désormais au centre de la démarche scientifique; le cœur des muséums palpite alors alternativement entre la diffusion d'un savoir universel issu de l'encyclopédisme et une expertise naturaliste taxonomique hyperspécialisée.

Les sociétés savantes d'Histoire naturelle ont joué un grand rôle dans la connaissance naturaliste : basées dans les écoles de médecines et les muséums d'Histoire naturelle, elles étudiaient et enrichissaient des fonds déjà réunis au siècle précédent par les amateurs des Lumières, constituant ainsi la base d'une connaissance indispensable à la compréhension du monde vivant. L'émergence des formations diplômantes au sein des universités rendit 
leur formation peu à peu désuète mais l'enrichissement des fonds se poursuivit au gré des personnalités et expertises de chacun et les données naturalistes liées aux observations via les collections s'accumulèrent. Après la Seconde Guerre mondiale, l'émergence des sciences moléculaires précipita certaines disciplines au rang des "obsolètes" et la donnée naturaliste semblait condamnée à ne plus intéresser que les naturalistes eux-mêmes. Les muséums poursuivirent pourtant, avec l'appui d'acteurs associatifs et d'amateurs éclairés, de nombreux projets de diffusion de cette connaissance du monde naturel.

\section{Internet et l'émergence des outils de diffusion culturelle de masse}

7 Les années 1980 voient l'essor de la numérisation des bases d'inventaires des musées. La diffusion de ces contenus experts reste alors confidentielle, pour des raisons techniques mais aussi en raison de l'absence d'un large outil de diffusion. Lors de la décennie suivante, de nombreux portails d'édition de bases documentaires voient enfin le jour, comme celui de l'inventaire (www.inventaire.culture.gouv.fr/Chemin_valo1.htm) ou la base Joconde (www.culture.gouv.fr/documentation/joconde/fr/pres.htm). En parallèle, la communication institutionnelle des musées se dote des premiers sites Internet comme l'exposition en ligne sur les requins du musée de paléontologie de Californie en 1993 ou le site du muséum de Londres l'année suivante qui devancent de plusieurs années l'essor d'Internet auprès du grand public, qui aura lieu véritablement au début des années 2000 .

Les coûts se réduisant alors de manière spectaculaire, une multiplication de bases informatiques thématisées de connaissances naturalistes a lieu (http:// collections.mnhn.fr/wiki/Wiki.jsp?page=Bases_Collections); les tentatives de regroupement des fonds des muséums au sein d'un portail national comme le GECO (gestion informatisée et valorisation par Internet des collections d'Histoire naturelle en région) préfigurent l'émergence des outils collaboratifs dédiés aux fonds naturalistes.

9 En parallèle, de nombreux observatoires de la biodiversité agrègent les données d'observation issues des acteurs d'un territoire sans aucun protocole commun de recueil et de diffusion des données (ce qui rendra leur interopérabilité plus difficile à mettre en œuvre) et sans avoir véritablement défini l'usage à moyen terme des données, notamment par le grand public qui reste alors bien éloigné de ces outils experts.

\section{L'émergence de l'open data et du crowdsourcing}

La France signe la charte de diffusion libre des données de biodiversité à l'aube des années 2000. La nécessité pour la communauté scientifique de réunir les éléments de connaissance dans des outils globalisés d'infrastructures de recherche comme le GBif (système mondial d'information sur la biodiversité) ou le SINP (Système d'Information sur la Nature et les Paysages) impose la standardisation des éléments à partager, ce que fera peu à peu l'INPN (Inventaire national du Patrimoine naturel). Qu'en est-il alors des muséums dans ce bouillonnement de l'open data naturaliste? En 2010, les muséums se trouvent alors en plein cœur de l'année de la biodiversité et s'interrogent sur leur rôle au sein de la stratégie nationale. Lieu d'éducation? Expert en sciences naturelles? Quelle stratégie pour les muséums au cœur d'un monde de la biodiversité en plein bouillonnement? Quel leadership dans le monde de la diffusion culturelle et scientifique? 
11 En parallèle, l'avènement $d u$ web 3.0 et du crowdsourcing constitue une révolution culturelle qui va permettre aux citoyens de véritablement participer à la production de la connaissance, notamment par le biais de la contribution. Ceci va créer pour les musées scientifiques de nouvelles opportunités d'être en relation avec les citoyens et d'affirmer leur compétence en matière de consolidation de la connaissance d'animateur de réseau.

\section{Les Nouveaux modes de production et de diffusion de la connaissance : atouts et risques pour les muséums}

Les muséums, détenteurs d'une expertise et d'un patrimoine culturel et scientifique important, peuvent enrichir considérablement la communauté numérique. Nous nous attacherons donc ici à proposer une analyse des atouts qui qualifient les muséums pour cette mission mais aussi les risques pour ceux-ci à s'investir dans la création et le pilotage de tels outils numériques contributifs.

\section{Enrichir le monde numérique, une extension naturelle des missions des muséums}

Internet et les médias sociaux sont un nouvel espace public et la rencontre du musée avec son public sur ce nouvel espace n'est qu'un prolongement logique d'une tendance plus large : l'élargissement de ses compétences (Besset, 2011). Historiquement, les institutions culturelles ont déplacé une partie de leur communication sur des sites Internet dès les années 1990, offrant ainsi à un public élargi une vitrine de leurs activités et la possibilité d'accéder à leurs contenus culturels, cœur même de leur mission de partage. L'essor du Web 2.0 dans les années 2000 a ouvert de nouveaux horizons en rendant le partage d'information entre usagers (avec ou sans l'institution) exponentiel, exacerbant le bouche-à-oreille et donc la notoriété des contenus et du musée lui-même ${ }^{1}$. Dès 2001, les premiers outils collaboratifs créés par des musées voient le jour et font l'objet de plusieurs publications lors de colloques dédiés, la possibilité pour le grand public d'interagir directement avec les contenus produits par les musées scientifiques étant vite envisagée comme une opportunité à saisir (www.archimuse.com). 
Depuis 2013, l'éléphante Eulalie tient un blog De mémoire d'éléphant sur la plateforme Echosciences Grenoble.

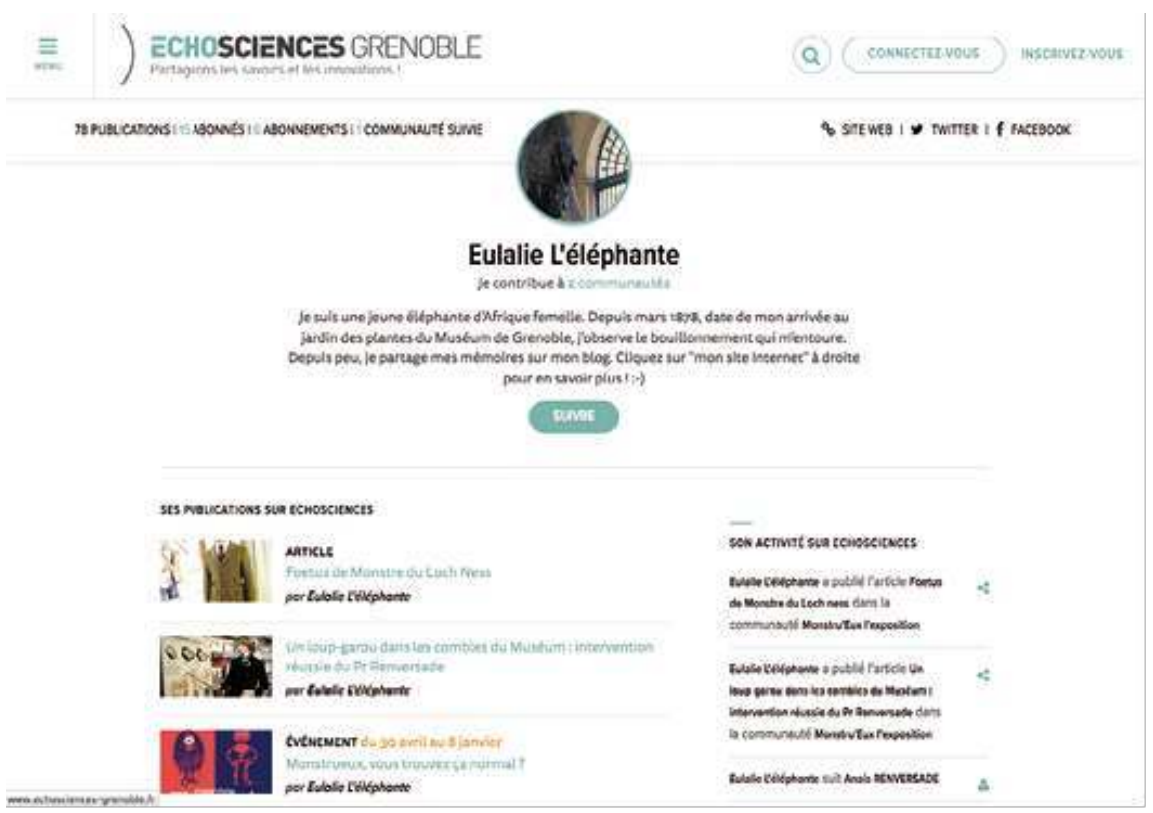

\section{Renforcer "l'estime de soi numérique"}

Partant du constat que la culture constitue une part de notre identité et que nous aimons échanger sur nos pratiques culturelles, les contenus libérés par les muséums renforcent l'identité positive de ceux qui s'en emparent, ce qui permet de comprendre le succès de journées comme "Ask a Curator" sur Twitter ou la "Museum week". Les réseaux sociaux sont d'ailleurs constitués de ces questions identitaires, prégnantes sur des réseaux comme Facebook. Les contenus liés à la culture ont de grandes chances d'y être diffusés puisqu'ils sont considérés comme "valorisants" ${ }^{2}$. Les contenus des musées, dégagés des logiques commerciales, y sont vus comme "authentiques" au regard des masses de contenus marketings diffusés sur Internet.

\section{Rapprocher la culture scientifique et les personnes qui ne s'y intéressent pas}

Les muséums sont des spécialistes avérés de la vulgarisation, de la "mise en accès" des connaissances car ils ont au cœur de leurs missions la "démocratisation culturelle". Or, l'enrichissement du monde numérique ne pourra se faire que si les contenus publiés sont perçus par les internautes comme accessibles, intéressants et valorisants. Les muséums doivent alors absolument éviter le piège de la publication de contenus trop complexes qui donnerait d'eux l'image d'un lieu "élitiste" et entrainerait le rejet d'une partie de la communauté numérique. Conquérir de nouveaux usagers via Internet est pourtant aujourd'hui un enjeu de démocratisation culturelle comme l'a démontré le muséum de Toulouse ; cela peut se faire de manière très directe en ouvrant par exemple un compte de photographies partagées ${ }^{3}$. 


\section{Donner une image moderne et dynamique de l'institution muséale}

16 La mise en commun d'éléments vulgarisés sur les outils numériques de partage peut être poussée plus loin, en créant véritablement un mode de communication très personnel avec ses usagers: "live twitt", reportage en ligne sur les coulisses, totémisation d'une "mascotte" comme par exemple l'éléphante Eulalie du muséum de Grenoble qui a ouvert un blog en 2013 sur la plateforme Echosciences Grenoble (www.echosciences-grenoble.fr/ communautes/de-memoire-d-elephant). Le musée devient alors plus proche de ses usagers, adoptant un ton moins "institutionnel" qu'il ne pourrait pas avoir sur des documents de communication officiels.

\section{Partager mais dans le respect de son identité propre}

17 La mutualisation des ressources économiques et intellectuelles ne peut se faire que si la mutualisation des acteurs la précède, soit par accord collectif des acteurs, soit sous la houlette d'un leader reconnu de tous. Les muséums ont été historiquement animateurs de nombreux réseaux de connaissances naturalistes ; ils bénéficient par ailleurs d'une image positive combinant pérennité comme les services d'archives et neutralité du service public. Ils sont donc à même de mener de telles "agrégations" d'intérêts divergents, et ce dans le respect de l'identité de chacun allant jusqu'à la contribution citoyenne.

\section{Le crowdsourcing ou génération d'un contenu original avec les utilisateurs}

18 En allant plus loin, il nous faut accepter que le contenu généré par les utilisateurs (photographies, articles, vidéos...) est une vraie richesse pour la communauté muséale comme c'est le cas au muséum de Toulouse ou dans le projet Musetrek qui permet aux utilisateurs de créer des "parcours culturels" en signalant des points d'intérêt ${ }^{4}$. Valoriser l'usager au même niveau que l'institution - par exemple par l'utilisation de concours mettant en avant les participations des internautes - est un signe fort de démocratisation culturelle. Ces outils sont de plus très intéressants en matière de médiation car ils rendent l'usager plus actif lors de sa visite s'il en a préparé en amont une partie.

Cela implique la désacralisation des contenus et donc de renoncer à sa position d'expert, et de perdre le contrôle sur les contenus produits. Les projets contributifs imposent une modération, adaptée, ce qui nécessite un investissement certain en ressources. Cette évolution implique aussi de créer un engagement fort et à moyen terme avec les contributeurs, comme il a été rappelé lors du congrès de l'AMCSTI 2015 (du participatif au contributif).

\section{Animer une communauté ou être à son service ?}

L'animation d'une communauté impose à son "community manager" de s'exposer. Il est impossible de mener de tels projets collaboratifs sans personnaliser l'institution notamment autour de son (ses) animateur(s). C'est là un changement de perspective qu'il nous faut pouvoir assumer. Nous sommes ici loin du "viral", du "bruit" permanent des "causeries" des réseaux sociaux ; l'investissement de chaque membre de la communauté 
se fera à l'aune de celui du musée, bien souvent au-delà de son animateur (validation de contenus proposés par l'équipe scientifique, propositions d'articles par l'équipe du musée...).

Le premier risque est de ne pas donner un objectif aux contributeurs. Il faut donc définir une stratégie en amont comme pour tout projet éditorial. Tous ces contributeurs potentiels se voient donner une mission, c'est là que l'idée des sciences participatives prend tout son sens sous réserve de pouvoir pérenniser l'engagement des contributeurs comme il a été démontré au congrès 2015 de l'AMCSTI $^{5}$. Ainsi, ouvrir un simple compte contributif de photographies doit répondre à une stratégie établie en amont.

Le deuxième risque est tout simplement de perdre son temps : l'organisation de tels projets transversaux puis leur animation peut devenir chronophage si le projet stratégique du musée n'est pas clair. Paradoxalement, nous pouvons aussi beaucoup y gagner si les contributeurs deviennent des "e-amis" du musée : un réel engagement pour l'institution peut naître de ces contributions.

Le troisième risque, lié à la production partagée de contenus, est celui de la propriété intellectuelle. Paradoxalement, les muséums sont ici avantagés car leurs collections sont rarement soumises à des libéralités contraignantes comme le droit de reproduction des œuvres d'art.

\section{Quels outils choisir?}

Une fois le projet défini et l'équipe mobilisée, faut-il investir un outil existant ou en créer un ex nihilo? Cette dernière solution est plus lourde mais elle représente l'occasion de construire des partenariats forts et d'obtenir un vrai engagement des contributeurs qui ne se disperseront pas comme lorsqu'ils sont contactés via des réseaux déjà existants.

\section{Nature-Isere.fr : quand la stratégie numérique d'un muséum rencontre l'urgence écologique}

La stratégie numérique du muséum de Grenoble

Face à tous ces questionnements, le muséum de Grenoble a mené en 2012 avec l'entreprise Decalog une étude stratégique pour envisager quelles opportunités semblaient pertinentes dans l'optique d'une réorientation plus large de ses missions.

Cette étude Muséum et usages numériques a permis de comprendre les outils qu'il était pertinent de développer pour le muséum dans le cadre de ses missions éducatives. Trois types d'usages ont été identifiés qui correspondent à trois grandes familles d'outils (schémas 1 et 2) que le muséum s'est attaché à développer depuis 2013, chacun ayant sa propre temporalité dans son agrégation/diffusion d'information.

De cette réflexion globale sur le muséum physique, couplé au muséum numérique, plusieurs projets (outils et usages) ont donc été initiés :

29 - le projet Nature-Isère, un portail des collections sous Opacweb 7 (Mobydoc) ;

30 - l'investissement dans des projets de partage des contenus liés aux expositions en creative commons sur la plateforme Echosciences Grenoble (exposition Monstrueux, vous trouvez ça normal ? en 2016) ; 
31 - des outils de lien direct avec le public : l'ouverture d'une page Facebook pour témoigner de la vie du service et activer de petits jeux (reconnaissance de collections) mais aussi ouverture d'un compte twitter pour faire de la veille en matière d'état de la biosphère et pour faire du "live twitt" (clins d'œil en direct des évènements).

\section{Un département isérois en pointe sur la question de la biodiversité}

En parallèle, des programmes scientifiques internationaux ont vu le jour dans le but de produire un état mondial de la biodiversité. La France a ratifié la charte de l'open data biodiversité dès 2001, et créé pour ce faire GBiff France (www.gbif.fr/). Face à la crise écologique, la création compulsive de bases de données régionales et départementales tels les observatoires de la biodiversité a multiplié les portails de data dans les années $2000^{6}$. La stratégie nationale de la biodiversité en 2010 a d'ailleurs ouvert une période de "frénésie" en matière de compilation de données. Le Conseil général de l'Isère, actif sur le plan de la biologie de la conservation, constate lui-même en 2008 lors d'un important séminaire dédié à la biodiversité l'absence de mise en commun des éléments de connaissance de son réseau. Un premier projet de plateforme régionale dédiée aux invertébrés échouera même pour avoir été trop spécialisé là où les acteurs de la botanique, portés par les deux conservatoires nationaux de la région, ont réussi à développer le pôle régional d'information Flore et Habitats (www.pifh.fr/pifhcms/ index.php).

En parallèle, diverses communautés d'observateurs ont vu le jour (comme www.fauneisere.org/) portées par des outils développés à la fin des années 2000, sous licence libre, mais forts complexes, spécialisées dans les données, pour et par des experts. En parallèle, de grands programmes de sciences participatives se multiplient, rendant complexes les collaborations entre les musées et ces outils car il y a foison de propositions. L'identité locale n'y est alors pas encore bien visible, freinant certaines ardeurs à s'investir dans un "grand tout" sans réelle valorisation des acteurs (ce qui a changé depuis: http:// sauvagesdemarue.mnhn.fr/sauvages-de-ma-ville/sauvages-de-ma-metro) mais aussi parce que ces observations portaient sur des espèces communes.

Il nous fallait réunir ces deux logiques, et tous les acteurs des mondes économique, éducatif, citoyen et scientifique.

Tout d'abord il nous a fallu convaincre quarante acteurs locaux - dont les acteurs institutionnels comme le Département de l'Isère (cent-vingt espaces naturels sensibles) ou la ville de Grenoble - de verser leurs données sur faune-isere (Biolovision). Ensuite, profitant du travail de standardisation des données entomologiques mené avec Pascal Dupont du Muséum national d'Histoire naturelle, nous avons entamé des discussions au niveau départemental sur les enjeux du partage de la connaissance au-delà de la donnée. Nous souhaitions aller plus loin que les outils existants, "experts" rappelons-le, pour pouvoir partager nos connaissances et échanger sur nos pratiques, tout en invitant des citoyens à observer certaines espèces pour augmenter la pression d'observations naturalistes sur des zones précises. Ensuite nous souhaitions que toutes ces informations soient éditoriales pour créer un "fil de l'eau" sympathique autour de la nature mais aussi de tous ceux qui y travaillent, en vivent, l'étudient.

Après de nombreux échanges avec les acteurs et accompagnés par une assistance à maîtrise d'ouvrage spécialisée, nous avons établi durant un an un cahier des charges 
détaillé : nature-isère.fr, espace contributif éditorialisé de découverte et de partage autour de la nature en Isère était né.

Schéma 1 : les trois sphères d'usages

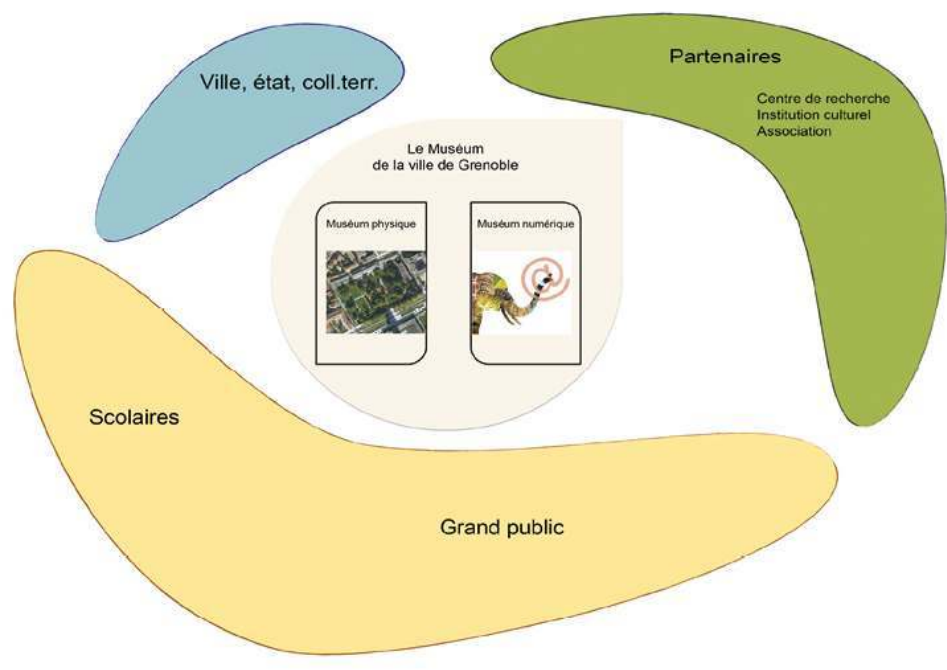

(c) Decalog/Muséum de Grenoble

Schéma 2 : les grandes familles d'outils

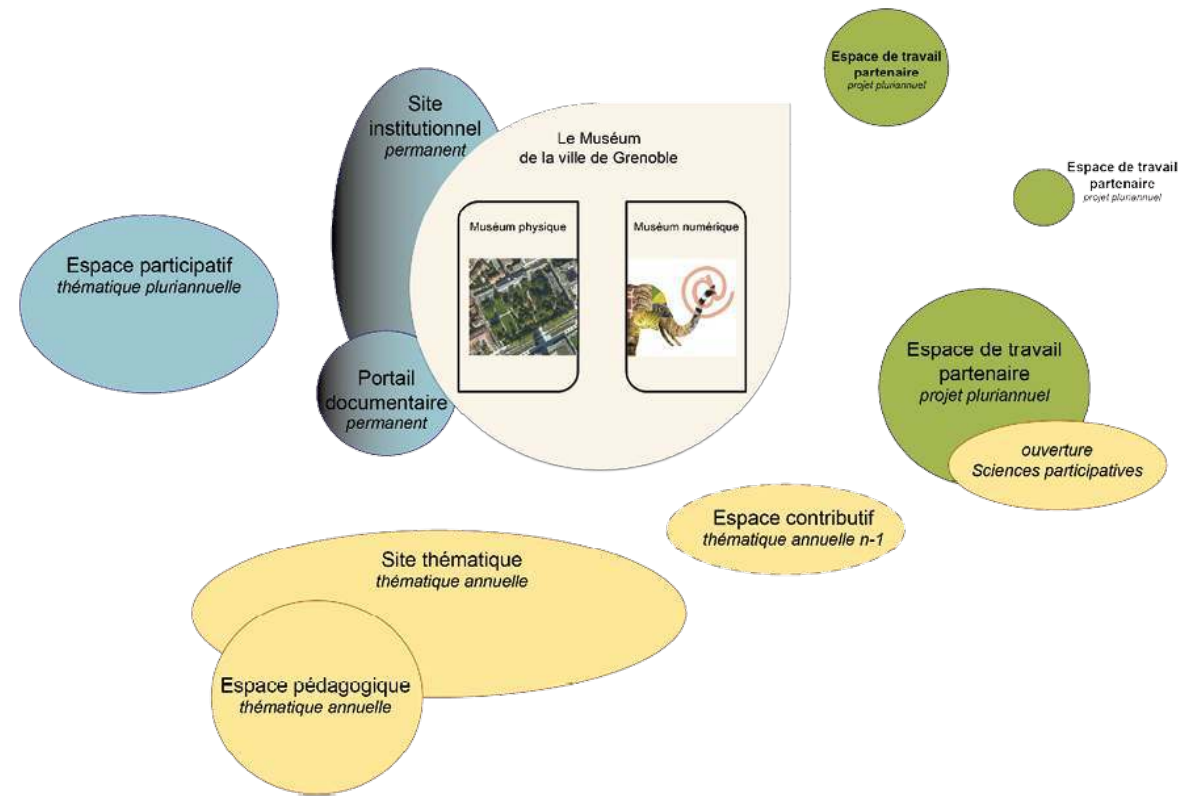

(C) Decalog/Muséum de Grenoble

\section{Le rôle du muséum de Grenoble dans le projet}

Réunir autant d'acteurs dans le respect des objectifs de chacun n'aurait pas été possible si le Département de l'Isère n'avait pas mis en œuvre depuis plus de quinze ans une 
stratégie collaborative de terrain avec tous ces acteurs en amont. Ces réunions régulières de coordination des divers schémas départementaux (faune, SDENS...) sont le terreau propice au développement de telles ambitions collaboratives. Par ailleurs, le muséum de Grenoble reste impliqué dans plusieurs instances de la biologie de la conservation au niveau départemental, régional et national, ce qui lui a donné une vraie légitimité dans certains échanges avec les réseaux de l'observation naturaliste. Le "plus" du muséum est d'avoir clairement positionné son leadership sur la question de l'accessibilité au savoir en s'appropriant l'axe 1 de la stratégie départementale en matière de diffusion de la connaissance. Combiné à une réorientation stratégique sur les usages numériques, l'équipement a pris la place de coordinateur du projet (il en porte l'appel d'offre notamment). Le muséum l'a mené à son terme avec les deux autres fondateurs (Département de l'Isère, Ligue pour la protection des oiseaux Isère) pour juin 2016. Par ailleurs, le recrutement d'un chargé du muséum numérique pour l'animation de Nature.Isere.fr a été concrétisé en mars 2016.

\section{Conclusion}

Les projets en crowdsourcing peuvent avoir une réelle valeur pour le musée, en termes d'implication des citoyens dans leur relation avec le projet et ses porteurs, et également en termes d'enrichissement de la connaissance par agrégation d'observations et de documentation. Cela va dans le sens de l'évolution de la société qui réinterroge notre relation à la gouvernance et qui investit de nouveaux modes de production de la connaissance.

Nous y voyons aussi une opportunité stratégique: celle de réinvestir avec leadership (c'est-à-dire une autorité reconnue par les autres membres du groupe) les réseaux de la connaissance. En effet, neutre, pérenne, expert de la vulgarisation scientifique comme du pilotage de projets culturels, tout muséum d'Histoire naturelle peut articuler de telles mises en commun de manière stable. Si cela demande parfois de l'énergie pour réunir les acteurs dans la formalisation d'un projet commun, le résultat n'en est que plus puissant, surtout lorsque les citoyens s'en emparent.

\section{Nature-Isere.fr : un espace contributif de découverte et de partage pour toutes celles et ceux qui découvrent et partagent autour de la nature en Isère}

Le ton de Nature-Isère est positif et ouvert. C'est un espace qui se veut enthousiaste, bienveillant, stimulant et pluraliste : toutes les sensibilités s'y retrouvent. Nous invitons les contributeurs à partager leurs contenus dans cet esprit. L'idée est d'échanger sur les bonnes pratiques, mais aussi d'inviter les visiteurs à observer la nature, les espèces et à compléter la connaissance en Isère.

\section{Qu'y trouve-t-on?}

41 - une carte pour trouver en un clic les espèces proches de l'utilisateur, dans un rayon de 5 kilomètres ; 
42 - les échos des contributeurs (échanges de bonnes pratiques, témoignages, actualités...) ;

43 - des ressources, partagées par tous les contributeurs : rapports scientifiques, comptes rendus de sortie de terrain, dessins d'enfants, cartes commentées, bibliographie et webographie...;

44 - des actions de terrain référencées avec leurs acteurs et de nombreuses propositions en vue de s'investir pour la nature.

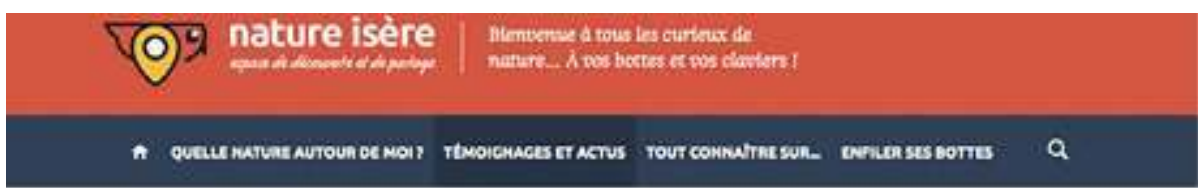

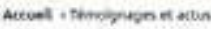

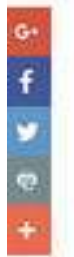

\section{TÉMOIGNAGES ET ACTUS}

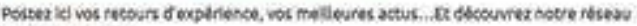
de contributeins.
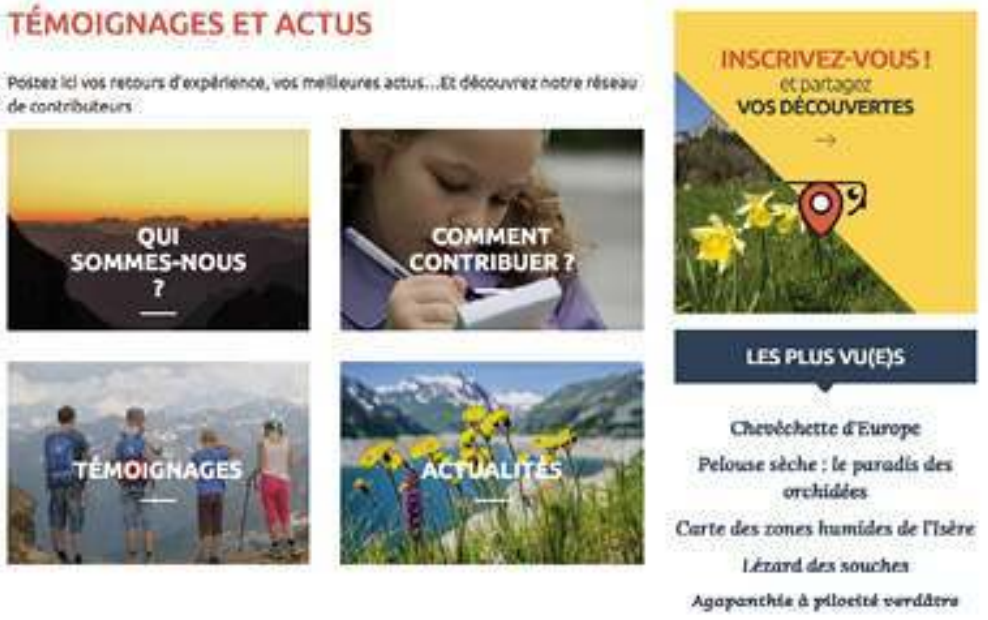

\section{Comment contribuer à Nature-Isère ?}

Deux types de contributions sont possibles :

46

- partager des données issues des observations sur le terrain. Ce type de contributions se fait via faune-isere.org ou son application Naturalist ;

47

- poster des documents, proposer une action, témoigner, commenter, soumettre une fiche espèce... 


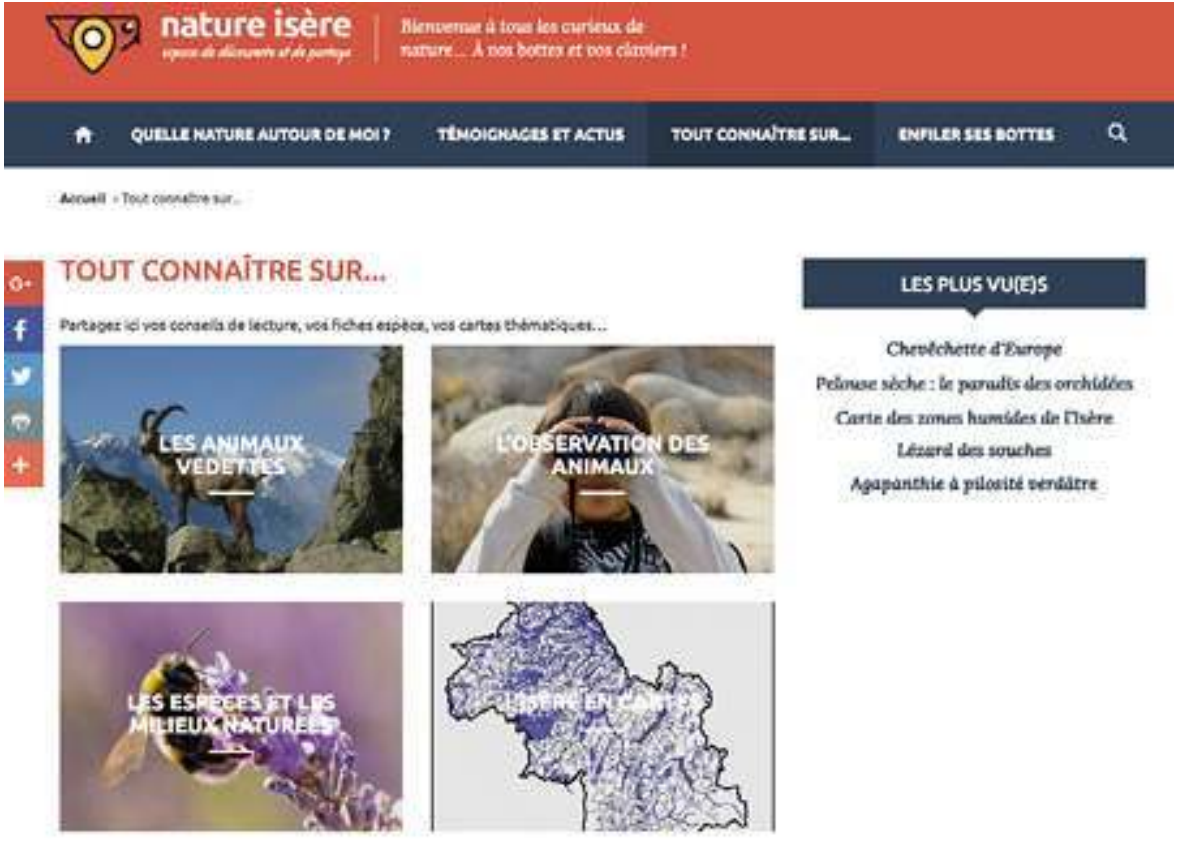

\section{Qui valide les contributions?}

48 Les contenus publics sont modérés en amont de leur publication, par le gestionnaire $d u$ site. En cas de litige, le comité éditorial arbitre les publications en lien avec la charte. Aucun sujet n'est a priori exclu mais Nature-Isère n'est pas un espace d'expression personnelle ou militante.

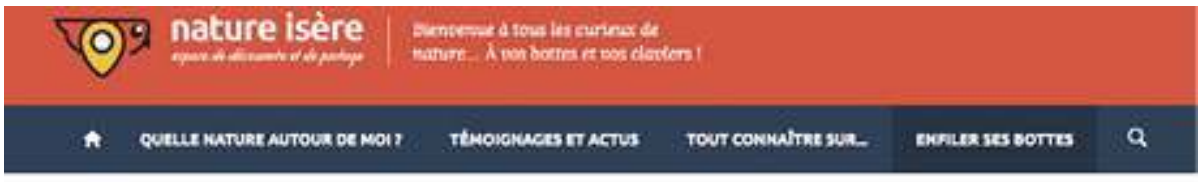

necoed i wist wisentes
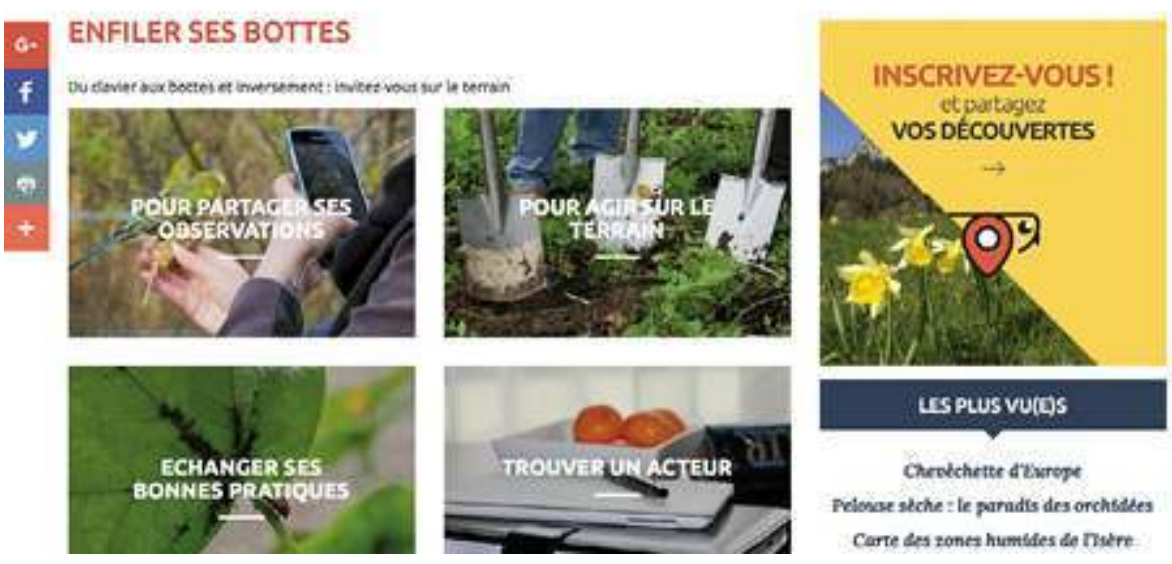

\section{Quelle gouvernance?}

49 La gouvernance de Nature-Isère s'organise autour de trois comités : 
50 - un comité éditorial qui réunit des personnes qualifiées dans le domaine du numérique, des sciences, de l'éducation, de l'environnement et du monde économique. Il est force de propositions pour l'animation éditoriale et rédactionnelle du site Nature-Isère, tant sur le fond que sur la forme des informations diffusées. Sa composition reflète la diversité des pratiques, des expériences et des avis de chacun ;

51 - un comité de pilotage qui suit l'avancée du projet et valide ses orientations stratégiques. Il est composé du gestionnaire de Nature-Isère, de l'entreprise prestataire et des trois partenaires fondateurs et porteurs du projet qui sont : le muséum de Grenoble, institution reconnue pour son action importante en matière de partage de la connaissance scientifique auprès de 100000 usagers chaque année et qui animera l'espace éditorial Nature-Isère en lien avec tous les contributeurs; le Conseil départemental de l'Isère; le réseau Faune-Isère animé par l'association LPO Isère qui fédère de nombreux acteurs observant la nature;

52 - un comité d'usagers qui regroupe une dizaine de personnes reflétant la diversité des utilisateurs et contributeurs de Nature-Isère qui donnent leur avis sur le fonctionnement de Nature-Isère.

\section{BIBLIOGRAPHIE}

Baert, J.-F., Pulh, M., Mencarelli, R., Graillot, L., Bourgeon-Renault, D. et Marteaux-Mencarelli, S. Quelles stratégies pour les musées sur internet? Actes de la $9^{\mathrm{e}}$ Journée de Recherche sur le emarketing, Paris : Université Panthéon Sorbonne, 2010.

Ben Sassi, M. Musée 2.0. De l'entrée du musée dans les réseaux à la remise en cause du public. Université Paris 1, juin 2008, www.scribd.com/doc/16361089/Musee-20-de-lentree-du-museedans-les-reseaux-a-la-remise-en-cause-du-public Besset, C. L'usage des médias sociaux par les musées : potentiel et réalisations. Majeure Médias Art et Création, juin 2011.

Estermann, B. Open data et crowdsourcing : un état des lieux du point de vue des musées, $L a$ Lettre de l'OCIM, $\mathrm{n}^{\circ}$ 162, novembre-décembre 2015, pp. 41-46.

London : Arts Council England. Digital Audiences engagement with arts and culture. MTM London, 2010. www.artscouncil.org.uk/media/uploads/doc/Digital_audiences_final.pdf

Stelmes, A. Les musées au prisme de la communication. Paris : CNRS Éditions, collection Hermès, $\mathrm{n}$ ${ }^{\circ} 61,2011,280 \mathrm{p}$.

Walsh, P. The Web and the unassailable voice (la désacralisation des contenus), www.archimuse.com/mw97/speak/walsh.htm 


\section{NOTES}

1. Utilisée pour la première fois en 2003 par Dale Dougherty, l'expression web 2.0 désigne une nouvelle manière d'envisager Internet, comme une plateforme ouverte, aux contenus accessibles et modifiables en continu par tous les usagers. C'est l'émergence des wikis, des blogs et bien sûr des plateformes collaboratives.

2. Claire Besset d'après Mehdizadeh, S. Self-Presentation 2.0: Narcissism and Self-Esteem on Facebook, Cyberpsychology, Behavior and Social Networking, vol.13, n4 , 2010, pp. 357-364.

3. Le compte du MuCem (www.slideshare.net/Dalbera/quelle-complmentarit-entre-le-site-webdune-exposition-et-flickr) a rencontré un grand succès ; le muséum de Grenoble va ouvrir un compte pour instagramers à l'occasion de son exposition Monstrueux vous trouvez ça normal ? réalisée en coproduction avec La Casemate, le CCSTI de Grenoble.

4. www.museum.toulouse.fr/reseaux et www.lelaboratoire.org/produits-musetrek.php (Le Laboratoire/Orange/Le Louvre).

5. Table ronde $\mathrm{Du}$ participatif au contributif www.youtube.com/watch? v=93gClICKzeg\&list=PLFEqtrEz60Aq8FN5dDw6CxLzRyWUKgmAv.

6. Par exemple celui du patrimoine naturel français http://inpn.mnhn.fr/accueil/index et celui de Wallonie http://biodiversite.wallonie.be/fr/accueil.html?IDC

\section{RÉSUMÉS}

Après avoir rappelé les différentes étapes historiques de la constitution et de la diffusion des connaissances naturalistes, l'auteur présente, à partir de l'expérience menée en Isère, les nouveaux modes de production de la connaissance à l'heure de la révolution du web 3.0 qui permettent aux citoyens de participer à la recherche par le biais de la contribution, avec une logique dans laquelle le rôle du muséum n'est plus central mais prescripteur et encadrant.

INDEX

Mots-clés : connaissance naturaliste, sciences citoyennes, muséum de Grenoble

\section{AUTEUR}

\section{CATHERINE GAUTHIER}

conservatrice du muséum de Grenoble catherine.gauthier@grenoble.fr 